\title{
Role of the long non-coding RNA LINC00052 in tumors (Review)
}

\author{
DONGMEI XIONG ${ }^{1}$, DAN WANG $^{2}$ and YANMENG CHEN ${ }^{3}$ \\ ${ }^{1}$ Early Childhood Health Research Innovation Team, Nursing School of Chongqing Medical and \\ Pharmaceutical College, Chongqing 401331; ${ }^{2}$ Clinical Laboratory, The People's Hospital of Rongchang, \\ Chongqing 402460; ${ }^{3}$ The Ministry of Education Key Laboratory of Laboratory Medical Diagnostics, \\ College of Laboratory Medicine, Chongqing Medical University, Chongqing 400016, P.R. China
}

Received August 25, 2020; Accepted February 4, 2021

DOI: $10.3892 / \mathrm{ol} .2021 .12577$

\begin{abstract}
Long intergenic non-protein coding RNA 52 (LINC00052) is a non-coding RNA with $>200$ nucleotides in length, which exerts important effects on several physiological and pathological processes of the human body. Recent studies have demonstrated that LINC00052 plays key roles in the tumorigenesis, progression and metastasis of multiple types of human cancer, including hepatocellular carcinoma, breast cancer, colorectal cancer, cervical carcinoma and gastric cancer. However, the associations between LINC00052 and these tumors remain unclear. The present review summarizes the biological functions of LINC00052 during the pathogenic process of certain tumors, and discusses its potential therapeutic targets.
\end{abstract}

\section{Contents}

1. Introduction

2. LINC00052 and HCC

3. LINC00052 and breast cancer

4. LINC00052 and CRC

5. LINC00052 and cervical carcinoma

Correspondence to: Dr Yanmeng Chen, The Ministry of Education Key Laboratory of Laboratory Medical Diagnostics, College of Laboratory Medicine, Chongqing Medical University, 1 Yixueyuan Road, Yuzhong, Chongqing 400016, P.R. China

E-mail: cym690@hotmail.com

Abbreviations: lncRNA, long non-coding RNA; LINC00052, long intergenic non-protein coding RNA 52; HCC, hepatocellular carcinoma; CRC, colorectal cancer; GC, gastric carcinoma; NTRK3, neurotrophic receptor tyrosine kinase 3; EPB41L3, erythrocyte membrane protein band 4.1-like 3; SOX9, SRY-related HMG-box gene 9; TNBC, triple-negative breast cancer; ER, estrogen receptor; PR, progesterone receptor; EGFR, epidermal growth factor receptor; HER3, human epidermal growth factor receptor 3; CALCOCO1, calcium binding and coiled-coil domain 1; STAT3, signal transducer and activator of transcription 3; TSCC, tongue squamous cell carcinoma

Key words: LINC00052, tumor, biological function
6. LINC00052 and GC

7. LINC00052 and other cancers

8. Conclusions and perspectives

\section{Introduction}

Long non-coding RNAs (lncRNAs) are functional small RNA molecules that cannot encode proteins (1). Increasing evidence suggest that lncRNAs exert important effects on the physiological and pathological processes of the human body, primarily through transcriptional or post-transcriptional mechanisms $(2,3)$. Long intergenic non-protein coding RNA 52 (LINC00052, National Center for Biotechnology Information reference sequence: NR_026869.1) is 1,786 base pairs in length and is located on human chromosome 15 (4). Abnormal expression of LINC00052 has been observed in several tumors, such as liver cancer (5-7), breast cancer (8) and colorectal cancer (9). The current research on LINC00052 mainly focuses on the effect of LINC00052 on tumorigenesis, cancer progression and metastasis (Table I).

The effect of LINC00052 on tumors was first reported by Xiong et al (5) in 2016, with the help of the gene trapping technique (10). The capture plasmid pU21 was transfected into hepatocellular carcinoma (HCC) cells through a plasmid vector. Subsequently, several single cell clones (for example A1 and A2) were obtained. The wound healing and Transwell assays demonstrated that the migratory and invasive abilities of the pU21 plasmid-obtained monoclonal cells were enhanced in the A26 and A55 cell lines, and slightly weakened in the A18 and A28 cells lines compared with the controls (5). 5 'RACE-PCR results demonstrated that the gene trapped by pU21 in the A55 cell line was LINC00052 (5); functional tests demonstrated that LINC00052 has the ability to regulate the invasion and migration of HCC cells (5). Subsequently, several studies have reported that LINC00052 is strongly associated with the occurrence and development of several tumors, including breast cancer (8), colorectal cancer (CRC) (9), cervical carcinoma (11) and gastric carcinoma (GC) (12). However, there is no conclusive article on the association between LINC00052 and these tumors. The present review summarizes the biological functions of LINC00052 during the pathogenic process of certain tumors and discusses its potential therapeutic targets. 


\section{LINC00052 and HCC}

HCC is one of the most common types of cancer worldwide. Bray et al (13) reported that $\mathrm{HCC}$ ranked sixth among the most diagnosed cancers $(841,080$ new cases) and was the fourth leading cause of cancer-associated mortality (781,631 deaths) in 2018, among the 38 types of tumors evaluated in 185 countries.

It has been proven that LINC00052 inhibits the invasion and migration of HCC cells (5). Through in-depth investigation of the molecular mechanisms underlying the effects of LINC00052 on the development of HCC, three target genes of LINC00052 were identified: Neurotrophic receptor tyrosine kinase 3 (NTRK3) (5), erythrocyte membrane protein band 4.1-like 3 (EPB41L3) (6) and SRY-related HMG-box gene 9 (SOX9) (7).

NTRK3 is a type of neurotrophin receptor that plays an important role in the nervous system, and it may be involved in the initiation, progression and metastasis of several human tumors, such as breast, medullary thyroid, liver, colon, lung and prostate cancers (14-16). Xiong et al (5) demonstrated that LINC00052 inhibits cell proliferation, invasion and migration by upregulating NTRK3 expression through complementary base pairing with microRNA (miRNA/miR)-128 and miR-485-3p. This is a key regulatory mechanism through which lncRNAs regulate target gene expression in tumors by sponging miRNAs, thus reducing their regulatory effect on mRNAs (17) (Fig. 1).

Zhu et al (6) reported that LINC00052 directly acts on EPB41L3 to affect the occurrence and development of HCC by targeting miR-452-5p. EPB41L3, a membrane skeletal protein that belongs to the protein 4.1 family (18), functions as a tumor suppressor in different tumors, such as GC, non-small cell lung cancer and renal clear cell carcinoma (19-21). In addition, Zhu et al (6) confirmed that EPB41L3 is a downstream target gene of LINC00052 via microarray, reverse transcription-quantitative PCR and western blot analyses. miR-452-5p was predicted to have two binding sites in the sequence of LINC00052 through the bioinformatics databases: DIANA-LncBase, https://bigd.big.ac.cn; miRcode, http://www. mircode.org and BiBiServ, https://bibiserv.cebitec.uni-bielefeld. de. Furthermore, it was demonstrated that miR-452-5p can recover LINC00052 function in HCC cells and inhibit EPB41L3 expression by targeting the 3 '-untranslated region of miR-452-5p (Fig. 1). Thus, Zhu et al (6) uncovered a novel LINC00052/miR-452-5p/EPB41L3 regulatory network in HCC.

The third target gene of LINC00052 is SOX9. SOX9 is a member of the SOX transcription factor family, which is associated with early embryonic development (22). SOX9 expression is frequently upregulated, and it is characterized as an oncogene in different types of human cancer, including lung cancer, GC and HCC (23-25). Yan et al (7) reported that LINC00052 promotes miR-101-3p expression by enhancing its promoter activity, and that miR-101-3p can interact with SOX9 to affect the occurrence and development of HCC (Fig. 1).

\section{LINC00052 and breast cancer}

Breast cancer is the leading cause of cancer-associated mortality among women worldwide (26). Bray et al (13) reported $2,088,849$ new cases and 626,679 breast cancer-associated mortalities in 2018, and breast cancer ranked second among the 38 types of tumors investigated in 185 countries. Thus, several studies continue to persistently investigate the pathogenesis and pathogenic genes of breast cancer, in the hope to identify biomarkers associated with its early diagnosis and treatment (27-29). By using RNA isolation, RNA-seq and microarray, Muñoz-Galindo et al (29) identified 221 and 146 dysregulated IncRNAs after 12 and 20 days of three-dimensional culture in breast cancer cells, respectively. It was demonstrated that LINC00052 was the most extensively downregulated lncRNA at two time points: Decreased by 47 -fold on the 12th day and 69-fold on the 20th day. These results suggest that LINC00052 is a key target for intervention in tumor diagnosis and treatment.

Triple-negative breast cancer (TNBC), which is characterized by the lack of estrogen receptor (ER), progesterone receptor (PR) and human epidermal growth factor receptor 2 expression, is an extremely aggressive type of breast cancer (30). Lv et al (31) demonstrated that LINC00052 is markedly upregulated in TNBC tissues compared with non-TNBC tissues and may serve as a powerful indicator for diagnosis of TNBC by analyzing the result of the receiver operating characteristic curve (area under the curve, $0.823 ; 95 \%$ confidence interval, 0.637-1.000). These results suggest that LINC00052 may serve as a potential biomarker for differentiating TNBC samples from non-TNBC samples.

Human epidermal growth factor receptor 3 (HER3/ErbB3) is a member of the epidermal growth factor receptor (EGFR) family of tyrosine kinase receptors (8). Upregulated HER3 expression has been implicated in the development and progression of different types of cancer $(32,33)$. For example, Salameh et al (8) confirmed that LINC00052 promotes the proliferation of breast cancer cells by increasing HER3 signaling both in vitro and in vivo (Fig. 1). This suggests that LINC00052 may act as a potential biomarker for HER3-targeted cancer therapies.

\section{LINC00052 and CRC}

CRC is a common malignant gastrointestinal tumor (34). Bray et al (13) reported that CRC ranked third among the most diagnosed cancers and second in mortality rate among 38 types of tumors in 185 countries, with 1,096,601 new cancer cases and 551,369 mortalities recorded in 2018. Previous studies have confirmed that IncRNAs are implicated in the occurrence and development of CRC, such as H19 (35), glycolysis-associated lncRNA of colorectal cancer (36) and fez family zinc finger protein 1-antisense RNA 1 (37). Yu et al (9) reported that downregulation of LINC00052 expression promotes the proliferative, migratory and invasive abilities of CRC cells. It was also confirmed that LINC00052 affects the occurrence and development of CRC by modulating the expression of calcium binding and coiled-coil domain 1 (CALCOCO1) through interaction with miR-574-5p (Fig. 1). CALCOCO1 is located on chromosome 12p13 and its different domains can bind with glutamate receptor interacting protein 1 and $\beta$-catenin, thereby affecting cell division and apoptosis (38).

\section{LINC00052 and cervical carcinoma}

Epidemiological statistics indicate that cervical cancer was the fourth most common gynecological malignancy worldwide 
Table I. Targets of LINC00052 in tumors.

\begin{tabular}{llll}
\hline Tumor type & \multicolumn{1}{c}{ miRNA } & \multicolumn{1}{c}{ Target gene } & Function \\
\hline HCC & miR-128, miR-485-3p & NTRK3 & Cell invasion and migration \\
& miR-452-5p & EPB41L3 & Cell invasion and migration \\
& miR-101-3p & SOX9 & Cell proliferation and metastasis \\
Breast cancer & - & HER3/ErbB3 & Cell proliferation \\
CRC & miR-574-5p & CALCOCO1 & Cell metastasis \\
Cervical carcinoma & - & STAT3 & Cell metastasis and invasion \\
GC & - & Wnt/ 3 -catenin pathway & Cell proliferation and metastasis \\
HNSCC & miR-608 & EGFR & Cell proliferation, migration and invasion \\
Glioma & - & KLF6 & Cell proliferation, migration and invasion \\
\hline
\end{tabular}

LINC00052, long intergenic non-protein coding RNA 52; HCC, hepatocellular carcinoma; CRC, colorectal cancer; GC, gastric carcinoma; HNSCC, head and neck squamous cell carcinoma; miR, microRNA; NTRK3, neurotrophic receptor tyrosine kinase 3; EPB41L3, erythrocyte membrane protein band 4.1-like 3; SOX9, SRY-related HMG-box gene 9; HER3, human epidermal growth factor receptor 3; CALCOCO1, calcium binding and coiled-coil domain 1; STAT3, signal transducer and activator of transcription 3; EGFR, epidermal growth factor receptor; KLF6, kruppel-like factor 6; -, not available.

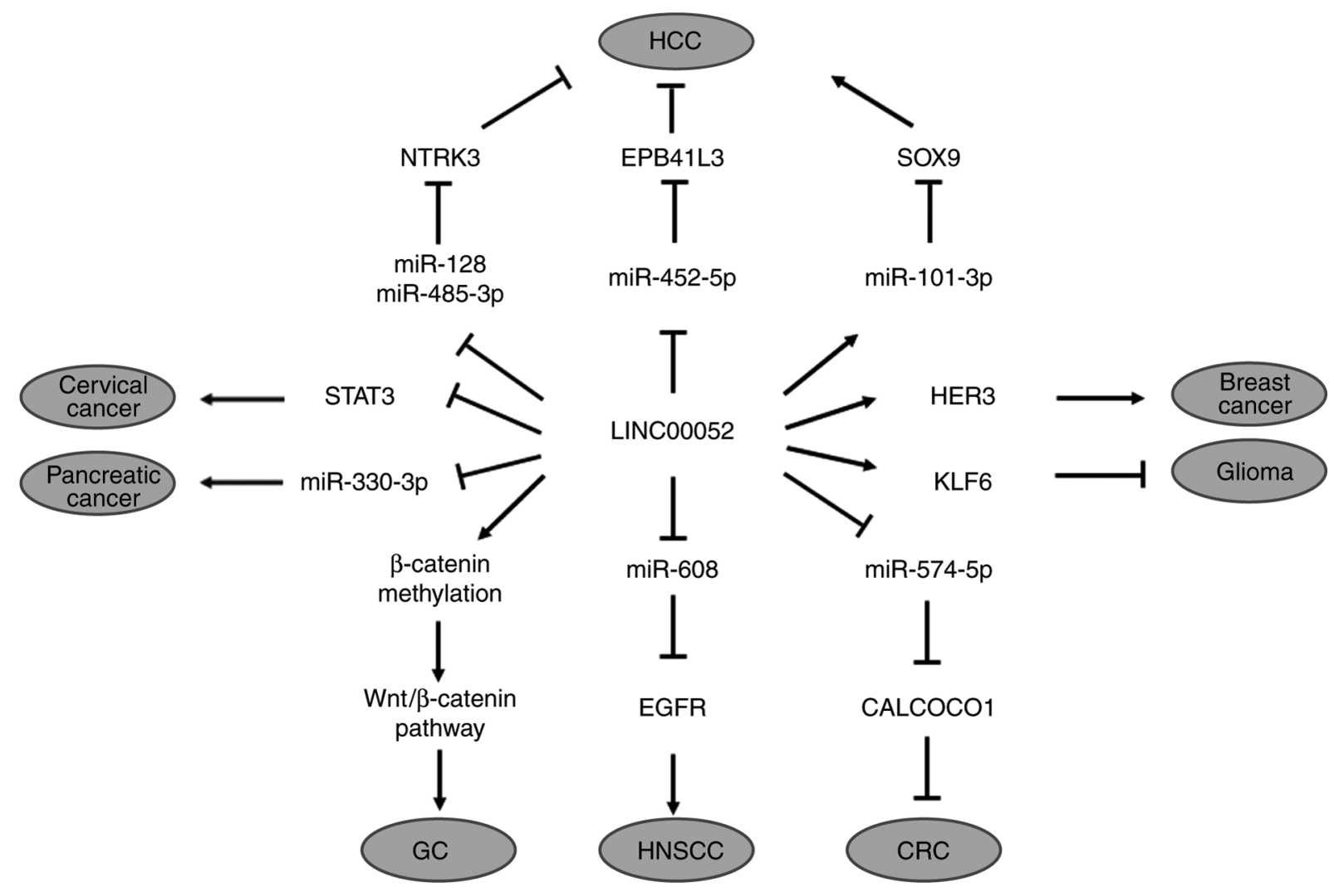

Figure 1. Schematic overview of role of LINC00052 in different tumors. LINC00052, long intergenic non-protein coding RNA 52; HCC, hepatocellular carcinoma; NTRK3, neurotrophic receptor tyrosine kinase 3; EPB41L3, erythrocyte membrane protein band 4.1-like 3; SOX9, SRY-related HMG-box gene 9; miR, microRNA; STAT3, signal transducer and activator of transcription 3; HER3, human epidermal growth factor receptor 3; KLF6, kruppel-like factor 6; EGFR, epidermal growth factor receptor; CALCOCO1, calcium binding and coiled-coil domain 1; GC, gastric carcinoma; HNSCC, head and neck squamous cell carcinoma; CRC, colorectal cancer.

in 2012 (39). Bray et al (13) reported that cervical carcinoma was the fourth most diagnosed cancer and the fourth leading cause of cancer-associated mortality in women among the 38 types of tumors in 185 countries, with 569,847 new cases and 311,365 mortalities reported in 2018. The use of cervical vaccines, early detection and timely treatment have decreased the incidence and mortality rates of cervical cancer (40). Increasing evidence suggest that lncRNAs serve as important regulators in the progression of cervical carcinoma (41). For example, lncRNA papillary thyroid carcinoma susceptibility candidate 3 has been demonstrated to suppress the invasion and proliferation of cervical cancer cells by regulating 
miR-574-5p expression (41), whereas lncRNA nuclear paraspeckle assembly transcript 1 regulates the proliferation and invasion of cervical cancer cells by targeting the PI3K/AKT pathway (42). Lin et al (11) demonstrated that LINC00052 expression is significantly downregulated in both cervical cancer tissues and cells. Overexpression of LINC00052 inhibits the proliferation, migration and invasion of cervical cancer cells. In addition, signal transducer and activator of transcription 3 (STAT3) protein expression is downregulated following overexpression of LINC00052 (11), suggesting that LINC00052 inhibits tumorigenesis of cervical cancer by suppressing the STAT3 pathway (Fig. 1).

\section{LINC00052 and GC}

GC is the fifth most common cancer and the third most common cause of cancer-associated mortality worldwide, with over one million estimated new cases and 782,685 deaths in $2018(13,43)$. Recent studies have reported that lncRNAs are associated with the occurrence and development of GC (44-46).

Shan et al (12) demonstrated that LINC00052 is highly expressed in GC tissues, which is associated with tumor progression and poor prognosis. The underlying molecular mechanisms of LINC00052 on the occurrence and development of GC were investigated, and the results demonstrated that LINC00052 maintains the stability of $\beta$-catenin by promoting $\beta$-catenin methylation to activate the $\mathrm{Wnt} / \beta$-catenin pathway (Fig. 1). This suggests that LINC00052 may promote the proliferation and metastasis of GC cells by activating the Wnt/ $\beta$-catenin pathway. However, some human GC cell lines (MGC-803, BGC-823 and SGC-7901) may be contaminated with HeLa cells (47), thus authenticated cell lines or animal models should be performed accordingly.

\section{LINC00052 and other cancers}

Tongue squamous cell carcinoma (TSCC) is the most common type of oral squamous cell carcinoma, which is characterized by marked invasiveness, early lymph node metastasis and poor prognosis $(48,49)$. To further investigate the molecular mechanism underlying the development and progression of TSCC, Zhang et al (50) identified a total of 1,867 mRNAs, 828 lncRNAs and 81 miRNAs that are aberrantly expressed in TSCC tissues compared with normal tissues via RNA sequence data processing, expression profile analysis and statistical analysis. It was predicted that LINC00052 is significantly associated with the overall survival rate of TSCC (50).

Pancreatic cancer is an aggressive malignant tumor with a poor prognosis (51). LINC00052 has been demonstrated to act as a tumor suppressor by negatively modulating miR-330-3p expression to affect the occurrence and progression of prostate cancer (52). In addition, LINC00052 may regulate the miR-608/EGFR axis to promote the progression of head and neck squamous cell carcinoma (53), and suppresses the proliferation, migration and invasion of glioma cells by upregulating kruppel-like factor 6 expression (54) (Fig. 1). Taken together, these results suggest that LINC00052 regulates a broad range of tumors.

\section{Conclusions and perspectives}

In the present review, the potential molecular mechanisms of action of LINC00052 in the pathological process of several tumors, such as HCC, breast cancer, CRC, prostate cancer and GC, were described (Table I). LINC00052 may represent a functional research hotspot in multiple tumors. LINC00052 can affect the tumor biological processes by regulating tumor cell proliferation, invasion, metastasis and migration. LINC00052 may serve as a miRNA sponge, competing with other genes for miRNA binding, and thus decrease mRNA transcription of targeted genes. However, further studies are required to validate the molecular mechanism of LINC00052. The tumorigenic mechanism of IncRNA is intricate and more information on the regulation of LINC00052 in response to malignant transformation is required to elucidate its complicated role in different tumors. In addition, the network of lncRNA/miRNA/target genes requires further investigation to determine the association between IncRNA expression and the occurrence and development of tumors. In conclusion, the present review summarizes the potential biomarkers or targets for novel therapeutic strategies.

\section{Acknowledgements}

Not applicable.

\section{Funding}

The present review was supported by the Chongqing Basic Research and Exploration Project (grant no. cstc2018jcyjAX0751).

\section{Availability of data and materials}

Not applicable.

\section{Authors' contributions}

DX, DW and YC were involved in the conception of the present review. DX drafted the initial manuscript. DW and YC critically revised the manuscript for important intellectual content. DX and YC confirmed the authenticity of all the raw data. All authors have read and approved the final manuscript.

\section{Ethics approval and consent to participate}

Not applicable.

\section{Patient consent for publication}

Not applicable.

\section{Competing interests}

The authors declare that they have no competing interests.

\section{References}

1. Li T, Mo X, Fu L, Xiao B and Guo J: Molecular mechanisms of long noncoding RNAs on gastric cancer. Oncotarget 7: 8601-8612, 2016.

2. Liz J and Esteller M: lncRNAs and microRNAs with a role in cancer development. Biochim Biophys Acta 1859: 169-176, 2016. 
3. Paraskevopoulou MD and Hatzigeorgiou AG: Analyzing miRNA-lncRNA interactions. Methods Mol Biol 1402: 271-286, 2016.

4. National Center for Biotechnology Information: Homo sapiens long intergenic non-protein coding RNA 52 (LINC00052), long non-coding RNA [DB/OL]. [2021-02-09]. https://www.ncbi.nlm. nih.gov/nuccore/223634020.

5. Xiong D, Sheng Y, Ding S, Chen J, Tan X, Zeng T, Qin D, Zhu L, Huang A and Tang H: LINC00052 regulates the expression of NTRK 3 by miR-128 and miR-485-3p to strengthen HCC cells invasion and migration. Oncotarget 7: 47593-47608, 2016.

6. Zhu L, Yang N, Chen J, Zeng T, Yan S, Liu Y, Yu G, Chen Q, Du G, Pan W, et al: LINC00052 upregulates EPB41L3 to inhibit migration and invasion of hepatocellular carcinoma by binding miR-452-5p. Oncotarget 8: 63724-63737, 2017.

7. Yan S, Shan X, Chen K, Liu Y, Yu G, Chen Q, Zeng T, Zhu L, Dang H, Chen F, et al: LINC00052/miR-101-3p axis inhibits cell proliferation and metastasis by targeting SOX9 in hepatocellular carcinoma. Gene 679: 138-149, 2018.

8. Salameh A, Fan X, Choi BK, Zhang S, Zhang N and An Z: HER3 and LINC00052 interplay promotes tumor growth in breast Cancer. Oncotarget 8: 6526-6539, 2017.

9. Yu GF, Xiong DM, Liu ZS, Li YG, Chen K and Tang H: Long noncoding RNA LINC00052 inhibits colorectal cancer metastasis by sponging microRNA-574-5p to modulate CALCOCO1 expression. J Cell Biochem 120: 17258-17272, 2019.

10. Tang H, Araki K, Li ZH and Yamamura K: Characterization of Ayu17-449 gene expression and resultant kidney pathology in a knockout mouse model. Transgenic Res 17: 599-608, 2008.

11. Lin J, Nong LL, Li MQ, Yang FC, Wang SH and Liu MJ: LINC00052 inhibits tumor growth, invasion and metastasis by repressing STAT3 in cervical carcinoma. Eur Rev Med Pharmacol Sci 23: 4673-4679, 2019.

12. Shan Y, Ying R, Jia Z, Kong W, Wu Y, Zheng S and Jin $\mathrm{H}$ LINC00052 promotes gastric cancer cell proliferation and metastasis via activating the Wnt/ $\beta$-catenin signaling pathway. Oncol Res 25: 1589-1599, 2017.

13. Bray F, Ferlay J, Soerjomataram I, Siegel RL, Torre LA and Jemal A: Global cancer statistics 2018: GLOBOCAN estimates of incidence and mortality worldwide for 36 cancers in 185 countries. CA Cancer J Clin 68: 394-424, 2018.

14. Jin W, Kim GM, Kim MS, Lim MH, Yun C, Jeong J, Nam JS and Kim SJ: TrkC plays an essential role in breast tumor growth and metastasis. Carcinogenesis 31: 1939-1947, 2010.

15. Genevois AL, Ichim G, Coissieux MM, Lambert MP, Lavial F, Goldschneider D, Jarrosson-Wuilleme L, Lepinasse F, Gouysse G, Herceg Z, et al: Dependence receptor TrkC is a putative colon cancer tumor suppressor. Proc Natl Acad Sci USA 110: 3017-3022, 2013

16. Satoh F, Mimata H, Nomura T, Fujita Y, Shin T, Sakamoto S, Hamada Y and Nomura Y: Autocrine expression of neurotrophins and their receptors in prostate cancer. Int J Urol 8: S28-S34, 2001.

17. Beermann J, Piccoli MT, Viereck J and Thum T: Non-coding RNAs in development and disease: Background, mechanisms, and therapeutic approaches. Physiol Rev 96: 1297-1325, 2016.

18. Wang Z, Zhang J, Ye M, Zhu M, Zhang B, Roy M, Liu J and An X: Tumor suppressor role of protein 4.1B/DAL-1. Cell Mol Life Sci 71: 4815-4830, 2014

19. Wang H, Xu M, Cui X, Liu Y, Zhang Y, Sui Y, Wang D, Peng L, Wang D and Yu J: Aberrant expression of the candidate tumor suppressor gene DAL-1 due to hypermethylation in gastric cancer. Sci Rep 6: 21755, 2016.

20. Zhang Y, Xu R, Li G, Xie X, Long J and Wang H: Loss of expression of the differentially expressed in adenocarcinoma of the lung (DAL-1) protein is associated with metastasis of non-small cell lung carcinoma cells. Tumour Biol 33: 1915-1925, 2012.

21. Yamada D, Kikuchi S, Williams YN, Sakurai-Yageta M, Masuda M, Maruyama T, Tomita K, Gutmann DH, Kakizoe T, Kitamura T, et al: Promoter hypermethylation of the potential tumor suppressor DAL-1/4.1B gene in renal clear cell carcinoma. Int J Cancer 118: 916-923, 2006.

22. Gao J, Zhang JY, Li YH and Ren F: Decreased expression of SOX9 indicates a better prognosis and inhibits the growth of glioma cells by inducing cell cycle arrest. Int J Clin Exp Pathol 8: 10130-10138, 2015.

23. Wang X, Ju Y, Zhou M, Liu X and Zhou C: Upregulation of SOX9 promotes cell proliferation, migration and invasion in lung adenocarcinoma. Oncol Lett 10: 990-994, 2015.
24. Choi YJ, Song JH, Yoon JH, Choi WS, Nam SW, Lee JY and Park WS: Aberrant expression of SOX9 is associated with gastrokine 1 inactivation in gastric cancers. Gastric Cancer 17: 247-254, 2014

25. Liu C, Liu L, Chen X, Cheng J, Zhang H, Shen J, Shan J, Xu Y, Yang Z, Lai M and Qian C: Sox9 regulates self-renewal and tumorigenicity by promoting symmetrical cell division of cancer stem cells in hepatocellular carcinoma. Hepatology 64: 117-129, 2016.

26. Fan L, Strasser KW, Li JJ, St Louis J, Finkelstein DM, Yu KD, Chen WQ, Shao ZM and Goss PE: Breast cancer in China. Lancet Oncol 15: e279-e289, 2014.

27. Zhao W, Geng D, Li S, Chen Z and Sun M: LncRNA HOTAIR influences cell growth, migration, invasion, and apoptosis via the miR-20a-5p/HMGA2 axis in breast cancer. Cancer Med 7: $842-855,2018$.

28. Dong H, Hu J, Zou K, Ye M, Chen Y, Wu C, Chen X and Han M: Activation of LncRNA TINCR by H3K27 acetylation promotes Trastuzumab resistance and epithelial-mesenchymal transition by targeting MicroRNA-125b in breast cancer. Mol Cancer 18: 3, 2019.

29. Muñoz-Galindo L, Melendez-Zajgla J, Pacheco-Fernández T, Rodriguez-Sosa M, Mandujano-Tinoco EA, Vazquez-Santillan K, Castro-Oropeza R, Lizarraga F, Sanchez-Lopez JM and Maldonado V: Changes in the transcriptome profile of breast cancer cells grown as spheroids. Biochem Biophys Res Commun 516: 1258-1264, 2019

30. Agrawal LS and Mayer IA: Platinum agents in the treatment of early-stage triple-negative breast cancer: Is it time to change practice? Clin Adv Hematol Oncol 12: 654-658, 2014

31. Lv M, Xu P, Wu Y, Huang L, Li W, Lv S, Wu X, Zeng X, Shen R, Jia X, et al: LncRNAs as new biomarkers to differentiate triple negative breast cancer from non-triple negative breast cancer. Oncotarget 7: 13047-13058, 2019.

32. Mujoo K, Choi BK, Huang Z, Zhang N and An Z: Regulation of ERBB3/HER3 signaling in cancer. Oncotarget 5: 10222-10236, 2014.

33. Baselga J and Swain SM: Novel anticancer targets: Revisiting ERBB2 and discovering ERBB3. Nat Rev Cancer 9: 463-475, 2009.

34. Wrobel P and Ahmed S: Current status of immunotherapy in metastatic colorectal cancer. Int J Colorectal Dis 34: 13-25, 2019.

35. Ren J, Ding L, Zhang D, Shi G, Xu Q, Shen S, Wang Y, Wang T and Hou Y: Carcinoma-associated fibroblasts promote the stemness and chemoresistance of colorectal cancer by transferring exosomal lncRNA H19. Theranostics 8: 3932-3948, 2018.

36. Tang J, Yan T, Bao Y, Shen C, Yu C, Zhu X, Tian X, Guo F, Liang Q, Liu Q, et al: LncRNA GLCC1 promotes colorectal carcinogenesis and glucose metabolism by stabilizing c-Myc. Nat Commun 10: 3499, 2019.

37. Bian Z, Zhang J, Li M, Feng Y, Wang X, Zhang J, Yao S, Jin G, Du J, Han W, et al: LncRNA-FEZF1-AS1 promotes tumor proliferation and metastasis in colorectal cancer by regulating PKM2 signaling. Cancer Res 24: 4808-4819, 2018.

38. Yang CK, Kim JH, Li H and Stallcup MR: Differential use of functional domains by coiled-coil coactivator in its synergistic coactivator function with beta-catenin or GRIP1. J Biol Chem 281: 3389-3397, 2006.

39. Ferlay J, Soerjomataram I, Dikshit R, Eser S, Mathers C, Rebelo M, Parkin DM, Forman D and Bray F: Cancer incidence and mortality worldwide: Sources, methods and major patterns in GLOBOCAN 2012. Int J Cancer 136: E359-E386, 2015.

40. Smith A, Baines N, Memon S, Fitzgerald N, Chadder J, Politis C, Nicholson E, Earle C and Bryant H: Moving toward the elimination of cervical cancer: Modelling the health and economic benefits of increasing uptake of human papillomavirus vaccines. Curr Oncol 26: 80-84, 2019.

41. Zhang M, Song Y and Yu L: LncRNA PTCSC3 suppressed cervical carcinoma cell invasion and proliferation via regulating miR-574-5p. Am J Transl Res 11: 7186-7194, 2019.

42. Guo HM, Yang SH, Zhao SZ, Li L, Yan MT and Fan MC: LncRNA NEAT1 regulates cervical carcinoma proliferation and invasion by targeting AKT/PI3K. Eur Rev Med Pharmacol Sci 22: 4090-4097, 2018

43. Smyth EC, Nilsson M, Grabsch HI, van Grieken NC and Lordick F: Gastric cancer. Lancet 396: 635-648, 2020.

44. Yu B, Chen X, Li J, Gu Q, Zhu Z, Li C, Su L and Liu B microRNA-29c inhibits cell proliferation by targeting NASP in human gastric cancer. BMC Cancer 17: 109, 2017. 
45. Arora N, Alsaied O, Dauer P, Majumder K, Modi S, Giri B, Dudeja V, Banerjee S, Von Hoff D and Saluja A: Downregulation of Spl by minnelide leads to decrease in HSP70 and decrease in tumor burden of gastric cancer. PLoS One 12: e0171827, 2017.

46. Tian J, Hu X, Gao W, Zhang J, Chen M, Zhang X, Ma J and Yuan H: Identification of the long non-coding RNA LET as a novel tumor suppressor in gastric cancer. Mol Med Rep 15: 2229-2234, 2017

47. Bian X, Yang Z, Feng H, Sun H and Liu Y: A combination of species identification and STR profiling identifies cross-contaminated cells from 482 human tumor cell lines. Sci Rep 7: 9774, 2017.

48. Abou-Elhamd KE and Habib TN: The role of chromosomal aberrations in premalignant and malignant lesions in head and neck squamous cell carcinoma. Eur Arch Otorhinolaryngol 265: 203-207, 2008

49. Zaman SU, Adeel M and Suhail A: Squamous cell carcinoma of oral tongue in young patients-A 10 years tertiary care experience. J Pak Med Assoc 66: 155-158, 2016.

50. Zhang S, Cao R, Li Q, Yao M, Chen Y and Zhou H: Comprehensive analysis of lncRNA-associated competing endogenous RNA network in tongue squamous cell carcinoma. PeerJ 7: e6397, 2019.
51. Bartel DP: MicroRNAs: Target recognition and regulatory functions. Cell 36: 215-233, 2009.

52. Xiong X, Shi Q, Yang X, Wang W and Tao J: LINC00052 functions as a tumor suppressor through negatively modulating miR-330-3p in pancreatic cancer. J Cell Physiol 234: 15619-15626, 2019.

53. Ouyang T, Zhang Y, Tang S and Wang Y: Long non-coding RNA LINC00052 regulates miR-608/EGFR axis to promote progression of head and neck squamous cell carcinoma. Exp Mol Pathol 111: 104321, 2019.

54. Xu CF, Liu P, Tan J and Hu DF: Long noncoding RNA LINC00052 suppressed the proliferation, migration and invasion of glioma cells by upregulating KLF6. Eur Rev Med Pharmacol Sci 23: 4822-4827, 2019.

This work is licensed under a Creative Commons Attribution-NonCommercial-NoDerivatives 4.0 International (CC BY-NC-ND 4.0) License. 